\title{
APROXIMACIÓN AL DEBATE SOBRE LA TRANSICIÓN SOLUTRENSE-MAGDALENIENSE EN LA CORNISA CANTÁBRICA (ESPAÑA)
}

\section{The issue on the Solutrean to Magdalenian transition in the Cantabrian Region (Spain)}

\author{
David Álvarez-Alonso ${ }^{1}$ y Álvaro Arrizabalaga ${ }^{2}$ \\ Recibido el 11 de marzo de 2013. Aceptado el 21 de mayo de 2013
}

\begin{abstract}
Resumen. En este trabajo realizamos un análisis historiográfico del horizonte transicional Solutrense-Magdaleniense en la región cantábrica (España), contextualizando el debate cantábrico en el contexto sobre la misma problemática en la región francesa. El objetivo principal es efectuar una exposición del estado actual de la investigación sobre el final del Solutrense en el norte de la Península lbérica.
\end{abstract}

Palabras clave: Badeguliense, región cantábrica, Magdaleniense arcaico, norte de España, Solutrense terminal.

\begin{abstract}
The main aim of this paper is to analyze, from a historiographic viewpoint, the transition from Solutrean to Magdalenian in the Cantabrian region (Spain), placing this issue in a broader context that includes central and southern France. We will present on it the current state of the arts about the end of Solutrean culture in northern Iberian Peninsula.
\end{abstract}

Keywords: Archaic Magdalenian, Badegoulian, Cantabrian region, North of Spain, terminal Solutrean.

\section{INTRODUCCIÓN}

El periodo transicional entre los tecnocomplejos Solutrense y Magdaleniense en la región cantábrica ha generado un intenso debate sobre su caracterización cultural y tecnoeconómica, amplificado tras la introducción en la discusión del concepto de Badeguliense cantábrico. El empleo de este concepto no comporta únicamente un problema terminológico o semántico, sino más bien antropológico, como indican L.G. Straus y G. A. Clark (2000).

Dos son los principales factores implicados en este debate. De un lado, el paso de una visión escalonada del desarrollo cultural humano (cada estadio cultural constituiria un peldaño), a otra más secuenciada, en la que los procesos de transición recaban una atención creciente (Camps y Chauhan 2009). Por otra parte, el proceso de adaptación de las sistematizaciones clásicas del Paleolítico en el marco regional francés a otros medios (como la región cantábrica), está aún lejos de concluir. En el fondo del debate se plantea si el proceso de tránsito, la continuidad entre poblaciones humanas distintas -o herederas unas de otras- $y$ las distintas manifestaciones culturales, pueden relacionarse con distintos procesos de adaptación al medio. Dentro de esta visión, debemos valorar aquella perspectiva que bascula entre

(1) Departamento de Prehistoria y Arqueología. UNED. Centro Asociado de Asturias. Avda. Jardín Botánico 1345.33203 Gijón. dalvarez@gijon.uned.es

(2) Departamento de Geografía, Prehistoria y Arqueología. Facultad de Letras. UPV-EHU. Francisco Tomás y Valiente, s/n. 01006 Vitoria. alvaro.arrizabalaga@ehu.es 
la dinámica de procesos autóctonos o la determinación de procesos exógenos que en su día desencadenaron el paso, desde ese constructo cultural que denominamos Solutrense, al Magdaleniense, a nivel regional.

En este trabajo trataremos de exponer el estado de la cuestión sobre este periodo tan determinante en el paso del Solutrense al Magdaleniense. ¿Realmente existe una transición, una solución de continuidad entre ambas? ¿Son los cambios tecnológicos manifestación de sucesiones o rupturas entre colectividades humanas? ¿Qué implicaciones tienen los cambios que se dan a nivel de cultura material en el poblamiento humano? No es nuestro objetivo resolver estas cuestiones, pero sin duda se trata de aspectos de suma importancia y que se hace necesario plantear en este debate sobre la transición o el paso de unas manifestaciones culturales, que definimos como solutrenses, a un mundo emergente que se ha definido como Magdaleniense y que se desarrollará a partir del Ultimo Máximo Glacial.

\section{EL FINAL DEL SOLUTRENSE Y LOS INICIOS DEL MAGDALENIENSE}

En primer lugar, podemos indicar que en la región cantábrica existe una aparente continuidad entre el Solutrense y el Magdaleniense inferior, ya apuntada desde los primeros estadios de la investigación paleolítica cantábrica, y de la que F. Jordá Cerdá dejó constancia tras una intensa labor de investigación centrada en varios niveles de distintos yacimientos asturianos (Jordá 1960). Hay que recordar que este investigador consideraba que el Magdaleniense cantábrico se iniciaba con el denominado Magdaleniense III, ocupando el Solutrense superior el espacio cronológico que en Francia correspondía al Magdaleniense I y II (Utrilla 2004) y considerando, por lo tanto, todo lo que se situaba por debajo del Magdaleniense III en la región, como Solutrense superior. Esta lectura, en la que el Magdaleniense cantábrico daba inicio con la fase III del esquema de Breuil, y donde los periodos 0 , I y II no estarian presentes, al contrario que en los yacimientos franceses, fue compartida entre otros por J. González Echegaray (1960), y pesó bastante en la investigación posterior, hasta que fue superada (González Echegaray y Barandiarán 1981). De esta manera, se consideraba la existencia de un desfase entre el final del Solutrense y el inicio del Magdaleniense, con respecto a Francia, que con el paso del tiempo se demostraría inexacta (Utrilla 2004). Se habian obviado, de este modo, las apreciaciones iniciales de Breuil, quien ya desde muy temprano indicó que el inicio del Magdaleniense en Cantabria (Santander, en la expresión de su tiempo) era contemporáneo del Magdaleniense I de Placard (Breuil y Saint-Périer 1927). En la misma dirección se expresó Vega del Sella al hablar de la Fase A del Magdaleniense cantábrico, situación que, hasta el análisis y esta- blecimiento del horizonte denominado "Rascaño 5" no fue confirmada, validando así las apreciaciones del Conde de la Vega del Sella y de Breuil (Utrilla 1989, 1996, 2004).

Uno de los principales problemas que tiene el análisis del periodo transicional Solutreo-Magdaleniense es el diferente enfoque realizado por los distintos investigadores, abordando la cuestión desde una perspectiva solutrense o magdaleniense, en función del interés dominante de cada especialista, lo cual ya supone un condicionamiento importante, como ha apuntado P. Utrilla (2004). De este modo, se ha ido produciendo una evolución en la consideración de los horizontes transicionales cantábricos (o finisolutrenses y magdalenienses iniciales), que ha ido paralela al desarrollo de la investigación en la región francesa. Las investigaciones sobre el Solutrense final en Francia y el inicio del Magdaleniense ya habían incorporado, desde los tiempos de A. Cheynier en primer lugar, y E. Vignard, más tarde, la consideración de un horizonte inicial para el Magdaleniense. Inicialmente, se denomina Protomagdaleniense, para posteriormente ser rebautizado como Badeguliense (Cheynier 1939, 1949; Vignard 1965). Aunque en un principio se consideró que este nuevo tecnocomplejo representaba el origen del Magdaleniense, el debate que se generó a partir de las secuencias francesas giró en torno a la consideración de un carácter "independiente" o "continuista" para el Badeguliense con respecto al Magdaleniense, ya que en aquel entonces estaba fuera de toda duda que suponía una clara ruptura con el anterior horizonte solutrense, a excepción de posturas como la de F. Trotignon (1984), que sí defendía tal filiación. Así, se fueron conformando diversas teorias sobre el origen del Badeguliense, su posible emplazamiento estratigráfico entre el Solutrense y el Magdaleniense, para finalmente ser considerado como un tecnocomplejo, que dará origen al Magdaleniense en el Occidente europeo (Bosselin 2000; Ducasse 2012). Para profundizar en estas cuestiones, que no son objeto principal de este trabajo, pueden consultarse los trabajos de Allain y Fritsch (1967), Bodu et al. (2007), Bosselin (2000), Bosselin y Djindjian (1997), Cretin (1996), Ducasse (2010, 2012), Ducasse y Langlais (2007) o Fourloubey (1998).

En los últimos años, algunos investigadores han incorporado al debate sobre la transición cantábrica la existencia de un tecnocomplejo badeguliense cantábrico (Bosselin 2000; Bosselin y Djindjian 1999), similar al horizonte contemporáneo francés, que es negado por unos autores (Corchón, 1994, 2005; Clark y Straus 2000) y aceptado con matices por otros (Utrilla 2004; Aura et al. 2012; Utrilla et al. 2012). Por ejemplo, mientras B. Bosselin y F. Djindjian defienden la existencia de un Badeguliense cantábrico a partir de la evidencia de industrias situadas entre el Solutrense y el Magdaleniense, caracterizadas por la rareza de laminitas de dorso, la abundancia de "útiles arcaicos" (escotaduras y denticulados, pero también raederas), la escasez de raclettes y una frecuencia media de raspadores y buriles (Bosselin y 
Djindjian 1999; Bosselin 2000), L. G. Straus y G. A. Clark (2000) desechan el uso del término, criticando vivamente a estos autores.

Podemos apuntar abiertamente que este debate ha caído algunas veces en una discusión semántica a propósito de la esencia del Badeguliense francés y las peculiaridades del Solutrense terminal cantábrico. G. Sauvet et alii (2008), en un interesante análisis sobre las relaciones territoriales en el Solutrense y Magdaleniense, consideran secundaria esta cuestión, ya que aceptan las diferencias que existen en este rango cronológico entre industrias que son diferentes del Solutrense superior y del Magdaleniense inferior, entendiendo que la controversia se centra, más en una cuestión terminológica, que de fondo. Destacan estos autores el hecho de que no parece probable que estas industrias, denominadas Badeguliense en Francia y objeto de debate y polémica en el Cantábrico, tengan tantas similitudes y hayan mostrado su presencia en un mismo horizonte cronológico, sin existir ningún tipo de relación entre ambas regiones (Sauvet et al. 2008:39).

\section{EL BADEGULIENSE FRANCÉS}

Es en Francia cuando, por vez primera, G. Vacher y E. Vignard (1964) individualizan el inicio del Magdaleniense, otorgándole la denominación de Badeguliense. A continuación, J. Allain (Allain y Fritsch 1967) añade que el Badeguliense sería el resultado de la llegada de nuevas poblaciones a finales del Solutrense superior, rompiendo con los clásicos paradigmas que partían de la unidad del Magdaleniense (Breuil 1937; Cheynier 1939). Para analizar este periodo, J. Allain procede a su división en dos etapas (inferior y superior) que coincidirian con el Magdaleniense 0 y I, definido por Sonneville-Bordes $(1960,1967)$ a partir de los datos de Lauguerie-Haute Est (Bordes, 1958). Como características principales, identifica algunas diferencias con respecto al Magdaleniense (datos obtenidos en las excavaciones del Abri Fritsch), como son la ausencia de hojitas de dorso, la importancia de las lascas como soporte lítico, la presencia de lo que denomina "raederas musteroides", la abundancia de buriles transversales sobre lasca o de raspadores de tipo "auriñaciense" con bordes astillados; igualmente, destaca la ausencia de raclettes en los niveles del Badeguliense inferior, mientras que en los del superior, es un elemento mucho más frecuente (Allain y Fritsch 1967; Ducasse 2012). La ruptura entre Badeguliense y Magdaleniense, así como la consideración de un Badeguliense en dos fases sucesivas (una sin raclettes y otra con raclettes), también fue seguida y desarrollada por B. Bosselin y F. Djindjian (1988), aceptando la línea de J. Allain, aunque posteriormente B. Bosselin (2000) afirme como posible que el Badeguliense evolucione hacia el Magdaleniense.
A partir de los trabajos de J. Allain, se generó un concepto de Badeguliense como un tecnocomplejo intrusivo, discordante con todo lo anterior y posterior, que sería fruto de la competencia entre grupos con un componente migratorio considerable, como recoge S. Ducasse (2012). Después de este primer planteamiento sobre el carácter y composición del Badeguliense, se llevaron a cabo diversos trabajos en fechas posteriores, pero todos ellos de indole tecnotipológica y sin llegar a entrar en el debate sobre la cuestión del origen y devenir del Badeguliense. Con la excepción de Fourloubey (1998), que propuso un discutido modelo de transición para el Badeguliense-Magdaleniense, partiendo de la diferenciación en fases o "facies" culturales, tanto con carácter sincrónico como diacrónico. Uno de los aspectos fundamentales a la hora de analizar el Badeguliense, ha sido siempre el de su filiación (Cretin 2007). De esta forma hubo partidarios de la independencia y ausencia de filiación entre Badeguliense y Magdaleniense (Allain, Cheynier, Trotignon, Vignard), del mismo modo que partidarios de considerar al Badeguliense como un tecnocomplejo a partir del cual evolucionaría el posterior Magdaleniense (Bazile, Bordes, Sacchi, Sonneville-Bordes) (Bosselin 2000).

A la hora de analizar este periodo, S. Ducasse (2012) define sus características esenciales, partiendo de la percepción de una gran variedad y adaptación en las estrategias tecnoeconómicas de los grupos badegulienses en las distintas regiones donde se localiza, y a partir de distintos tipos de materia prima (siempre de carácter local). Así, habla de un bajo y moderado grado de requerimiento, visible a través de los tipos, dimensiones, morfologias y calidad de las materias primas seleccionadas. También apunta que las lascas sustituyen a las láminas, siendo éste un marcador de la menor complejidad pero igual eficiencia. Este autor incide en la búsqueda de la efectividad técnica por parte de los badegulienses, que explotarian intensamente las materias primas locales gracias a la implementación de soluciones técnicas simples o flexibles, con la diversificación y ramificación de las cadenas operativas desarrolladas. No considera, por tanto, que se trate de una industria arcaizante, sino más bien de una adaptación a modos de producción más efectivos que los anteriores, basándose en la simplificación de los esquemas tecnológicos y, por tanto, con un resultado de apariencia "menos elaborada". Extremo que se acentúa por contraposición a los conjuntos del Solutrense superior y a los conjuntos laminares del Magdaleniense inferior.

En consecuencia, podemos interpretar que, en Francia, el debate sobre esta transición se desarrolló por una senda en la que se trató de individualizar este proceso, bajo el prisma de un tecnocomplejo badeguliense, bien como un tecnocomplejo intermedio, bien como un Magdaleniense arcaico o inicial. Uno de los principales problemas, al que se refiere $C$. Fourloubey (1998) es que, indistintamente, los investigadores se han referido a los mismos tecnocomplejos usando en unas 
ocasiones el término de Badeguliense y en otras el de Magdaleniense antiguo o inicial, cuando realmente se trataría de lo mismo. Por ello, este autor considera que en el debate hay cuestiones de fondo sobre la caracterización del periodo (que parece claro), y cuestiones secundarias, relativas a la terminología empleada, pero a las que se ha concedido excesiva importancia y han generado confusión.

Este último problema también se reproduce en la región cantábrica, aunque con el uso de otros términos. En la Península lbérica, en concreto en el Cantábrico, el debate discurrió hacia la definición de una fase arcaica o inicial para el Magdaleniense, denominada Rascaño 5, a partir de la identificación de unos caracteres bien individualizados en dicha cueva (sobre todo las denominadas azagayas tipo Placard). Dejando las cuestiones terminológicas aparte, está claro que en ambos casos se está planteando la identificación de un Magdaleniense arcaico o inicial, según unos sin una clara vinculación con el desarrollo posterior del Magdaleniense, aunque en los últimos años está quedando más patente la existencia de cierta vinculación planteada por muchos especialistas. También parece evidente la existencia de varias etapas dentro de este proceso, que dura unos dos mil años aproximadamente. Esta estructuración puede seguirse en Ducasse (2012), Utrilla (2004) y Aura et al. (2012).

Otra cuestión aparte, es el debate sobre la presencia de piezas solutrenses típicas en contextos que tecnotipológicamente son coherentes con un Magdaleniense arcaico o Badeguliense, o como quiera que prefiramos referirnos a él. ¿Se trata de intrusiones de índole postdeposicional o tafonómica? ¿son piezas seleccionadas intencionalmente e incorporadas al yacimiento en un contexto no Solutrense? ¿hay una pervivencia de cierto utillaje más allá de su pertenencia a su tecnocomplejo originario? Si aceptamos las teorías migratorias, que nos llevan a considerar la existencia de diversos grupos humanos coetáneos y culturalmente diferentes ¿serian estas piezas muestras de una aculturación? o más bien ¿serían fruto de un cambio tecnológico interno, dentro de los propios grupos solutrenses como respuesta, tanto a los cambios culturales importados por nuevos grupos, como a las nuevas necesidades de adaptación ante los cambios ambientales que se están produciendo?

Estas son preguntas difíciles de contestar y que tampoco constituyen un objetivo primordial en este trabajo de síntesis. Desde luego, la incorporación de los estudios y análisis de ADN sobre poblaciones del Paleolítico superior debe intensificarse (aunque los restos son limitados), puesto que esta línea de investigación está llamada a resolver muchos de los interrogantes que hoy en día se nos presentan como de difícil resolución. Los datos aportados por el ADN podrian posibi- litar nuevos planteamientos interpretativos, con novedosas propuestas de análisis a la hora de considerar los tecnocomplejos líticos de este periodo, sus filiaciones y su composición desde una perspectiva más antropológica, y no únicamente tecnotipológica, con todos los problemas y carencias que sabemos que el análisis tecnotipológico conlleva y que, lógicamente, asumimos siempre como punto de partida.

\section{LA TRANSICIÓN EN LA REGIÓN CANTÁBRICA}

En 1983, L. G. Straus manifestaba que el Solutrense superior estaba caracterizado por una gran variedad y diversidad en la frecuencia de aparición de utillaje, considerando notables diferencias entre los conjuntos con hojitas y buriles, y los conjuntos en los que predominan los denticulados, escotaduras y raederas. Este autor consideraba que esta situación era fruto de una gran variabilidad, llegando a decir que "no hay un conjunto-modelo solutrense". Puede que estas impresiones, en la época en la que se efectuaron (década de 1970 y primeros 80) no estuvieran sino manifestando una complejidad y diversidad tecnocultural (Straus 1983), que hoy en día vemos como mucho más variada y que sin lugar a dudas, parece transcender más allá de los propios límites establecidos en su momento para el Solutrense superior cantábrico.

El principal problema a la hora de valorar la diversidad y complejidad existente entre el Solutrense superior y el Magdaleniense inferior, en principio, respondia a la escasez de datos procedentes de excavaciones sistemáticas realizadas en época moderna, con completas analíticas y datos paleoambientales y/o cronológicos. La ausencia de un contexto cronoestratigráfico claro, el hecho de basarse en niveles y materiales procedentes de excavaciones antiguas, los abundantes problemas tafonómicos existentes 0 las fases erosivas identificadas en los niveles de transición " hicieron complejo analizar el proceso de tránsito entre el Solutrense y el Magdaleniense. Una cuestión clave para entender la investigación desde una perspectiva historiográfica es el hecho de que muchos niveles fueron estudiados en un primer momento bajo un "prisma solutrense", para posteriormente analizarse críticamente bajo una amplia perspectiva, y ser más tarde recalificados o desgajados del "paquete cronoestratigráfico solutrense".

Con respecto a la identificación del final del Solutrense, M. a S. Corchón (1981) habla de un "Solutrense terminal" para referirse a los cambios apreciados en el techo de la secuencia solutrense de Las Caldas. Este término es mati-

(1) En el monográfico El Solutrense en la Península Ibérica (1994), publicado como no 1 de la revista Férvedes, pueden hallarse abundantes referencias a los problemas apuntados, al igual que en Utrilla (2004) o en Aura et al. (2012). 
zado posteriormente por M. de la Rasilla (1989), que considera impreciso hablar de "terminal" puesto que se trataba de una zona de excavación limitada, había escasos datos y debian recordarse algunas explicaciones que hablaban de la distinta funcionalidad y representación tecnotipológica de los yacimientos solutrenses (Straus 1983). En cambio, este investigador considera más apropiado hablar de un proceso de desolutreanización y habla, por tanto, de "Solutrense superior desolutreanizado" (Rasilla 1989, 1994). Este autor también plantea la posibilidad de que los niveles protagonistas de esta problemática, se correspondan realmente con etapas transicionales. Por esta razón, considera oportuno hablar también de proceso de "magdalenización", indicando que se trataría de un proceso gradual, no brusco, desde el Solutrense, hacia el Magdaleniense, realizado por los mismos grupos, y poniendo como ejemplo del mismo los niveles 15-17 de La Riera. Considera, de este modo, que existe cierta aculturación o asimilación (Rasilla 1989). Queda patente que existen una serie de niveles e industrias de difícil adscripción al Solutrense superior o al Magdaleniense inferior, y a la vez unidos estrechamente a ambos horizontes (sobre todo al Magdaleniense inferior suprayacente), en lo que en algunos yacimientos aparece como una continuidad estratigráfica, sin fisuras ni rupturas. Por otra parte, las similitudes entre el Solutrense superior y el Badeguliense cantábrico / Magdaleniense arcaico han permitido pensar a algunos investigadores en un proceso de transición, antes que en una sustitución (Aura et al. 2012), siguiendo en la línea de planteamientos similares que apuntaban a un largo y lento proceso de magdalenización (Rasilla 1989, 1994).

En el interesante debate sobre la caracterización de los niveles en litigio, resulta trascendente la incorporación de las líneas de investigación seguidas en el Suroeste francés, a la luz de la estrecha relación de la Dordoña, Aquitania o Pirineos con el Paleolítico superior cantábrico. De este modo, y a la luz del debate sobre el Badeguliense francés, se introduce primero este término en el ámbito levantino de la cueva de Parpalló (Aura 1988, 1995, 2007), para luego plantearse su existencia en el Cantábrico (Bosselin y Djindjian 1999; Bosselin 2000), a costa de antiguos niveles asignados al Solutrense superior 0 al Magdaleniense arcaico. Con respecto al Badeguliense del Levante, recientemente se ha planteado el gran paralelismo existente entre el Badeguliense de Parpalló y el Magdaleniense arcaico cantábrico, considerándose un mismo horizonte cultural (Aura et al. 2012). También, Sauvet et al. (2008) han considerado la existencia de un horizonte bien diferenciado del Solutrense superior y del Magdaleniense en la horquilla 18500-16500 BP, que se correspondería con los clásicos Magdalenienses 0 y I, y que recibe el nombre de Badeguliense 0, o en el caso cantábrico, Magdaleniense arcaico. Para estos autores, la presencia de este horizonte en el Cantábrico, muestra las afinidades y relaciones existentes con el fenómeno contemporáneo que se produce en Aquitania. Vemos así cómo los diferentes puntos de vista, en cuanto a la aceptación de un horizonte Badeguliense cantábrico, deben ser matizados y expuestos correctamente en el debate historiográfico sobre el fin del Solutrense y el inicio del Magdaleniense.

\section{LA CARACTERIZACIÓN DE LAS DISTINTAS FASES Y NIVELES TRANSICIONALES CANTÁBRICOS}

Uno de los puntos fundamentales a la hora de afrontar este debate en la región cantábrica, es el análisis de las distintas fases descritas para este periodo transicional. Por ello, es preciso intentar realizar una aproximación a su trasfondo tecnocultural, pero también conceptual e historiográfico. Hay que entender que el contexto es el mismo, el problema es uno, y lo que varía en cada caso es el enfoque con el que se ha realizado la aproximación interpretativa del registro. De este modo, describimos a continuación la sucesión de las fases y características tipológicas planteadas para los niveles encuadrados en el proceso de transición solutrensemagdaleniense en la región cantábrica.

\subsection{Solutrense terminal (Corchón 1981)}

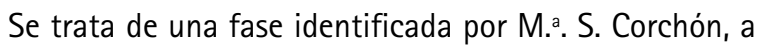
partir de sus excavaciones en la cueva de Las Caldas. Las caracteristicas principales, consisten en una rarificación del utiIlaje solutrense, aumento de la cuarcita como materia prima, bajo índice laminar y disminución del tamaño del utillaje. M. de la Rasilla (2003) considera que este Solutrense es terminal en Las Caldas (el final del Solutrense en esa secuencia), pero no tiene por qué ser representante del final del proceso denominado por él como "desolutreanización", considerando que solo plasmaría una situación estratigráfica.

En cualquier caso, y sin entrar a valorar si representa 0 no el último vestigio solutrense del Cantábrico, la presencia de este "Solutrense terminal" en Las Caldas es indicativa de que, al final del periodo solutrense se empiezan a producir una serie de cambios, que sin duda, finalizarán con la aparición del Magdaleniense.

\subsection{Solutrense desolutreanizado (Rasilla 1989, 1994, 2003; Straus y Clark 2000)}

M. de la Rasilla aborda el proceso de tránsito del Solutrense al Magdaleniense, desde una perspectiva continuista, en la que se produciria no sólo una "desolutreanización", sino también un paulatino proceso de "magdalenización". Este Solutrense desolutreanizado se caracterizaría por la importancia de las lascas, con restricción de las hojas a ciertos utensilios, baja laminaridad, 
simplificación del modelo tipológico, creciente importancia de la lámina de dorso y rarificación del utillaje solutrense. M. de la Rasilla incluye los niveles 6-3 de Las Caldas y 9-14 de La Riera en este proceso, apuntando la posibilidad de que sean etapas transicionales; por otra parte, considera que el proceso de magdalenización se hace evidente en los niveles 15-17 de La Riera, considerando el último (n.17) como Magdaleniense (Rasilla 1989). Posteriormente, el mismo autor (Rasilla 1994), al referirse a este periodo indica que las laminitas de dorso van perdiendo poco a poco su depurada técnica solutrense para convertirse en laminitas fabricadas en cualquier soporte mediante retoque. En esta segunda publicación ya incluye los niveles 15-17 de La Riera en el Magdaleniense, y hace una relación de los niveles que deben encuadrarse en el Solutrense superior desolutreanizado: Morín, Cova Rosa, Aitzbitarte IV, Buxu, Chufín, Llonín IV, Amalda IV, La Riera 9-14 y Las Caldas 6-3 (Rasilla 1994). Este proceso lo sitúa entre el 18000 BP y el 17000 BP, a finales del inter Laugerie/Lascaux e inicios del Lascaux (Rasilla 1989, 1994). Como respuesta a la interpretación de los niveles solutrenses de La Riera, recalificados como Badeguliense por B. Bosselin y F. Djindjian (1999), L. G. Straus y G. A. Clark (2000), responsables de la excavación de dicho yacimiento (Straus y Clark, 1986) niegan tal atribución, rechazando la existencia del Badeguliense en la región cantábrica y considerando que en realidad lo que existe entre 18000 y 17000 BP en la región es un proceso de desolutreanización, como parte de una evolución interna, desechando así cualquier posible influencia migratoria de grupos con nuevas tecnologías.

\subsection{Badeguliense cantábrico (Bosselin y Djindjian 1999; Bosselin 2000)}

Según estos autores, en el Cantábrico solo sería reconocible lo que han denominado como Badeguliense Tipo $A$, equiparable al Badeguliense inferior en Francia, y que estaría presente en los yacimientos de La Riera 8-16 (clasificado como Solutrense terminal por L. G. Straus y G. A. Clark 1986), Las Caldas 6-4, El Cierro 4, Cova Rosa 2-3, La Lloseta 10, Cueto de la Mina D, El Castillo, Rascaño 5 (Bosselin y Djindjian 1999).

Estos autores se basan en la secuencia de La Riera para identificar las características que definirian este tecnocomplejo en el Cantábrico, y que se resumen en: abundancia de útiles "arcaicos" (denticulados y escotaduras), frecuencia media de raspadores y buriles, desarrollo de láminas retocadas, escasas raclettes y poca representación de las laminitas de dorso y la presencia esporádica de útiles solutrenses; en los niveles 15-16 de La Riera se destaca la sustitución parcial de las escotaduras y denticulados por piezas astilladas, raederas, raclettes y una creciente presencia de laminitas de dorso (Bosselin y Djindjian 1999).

Uno de los aspectos más criticados de este planteamiento no es la caracterización del periodo, sino más bien un matiz de la interpretación del significado de este $\mathrm{Ba}$ deguliense cantábrico en la secuencia del Paleolítico superior de la región. Estos autores (Bosselin y Djindjian 1999) consideran que esta intergradación entre el Solutrense y el Magdaleniense se explica como un nuevo grupo cultural que irrumpe en la región, dando por lo tanto una explicación migratoria al problema, algo que ha sido fuertemente criticado por L. G. Straus y G. A. Clark (2000); aunque estos últimos no niegan que esto no haya sucedido en Aquitania. Por otra parte, B. Bosselin (2000) deja patente su consideración de la existencia de filiación entre el Badeguliense y el Magdaleniense inferior en el Cantábrico, además de apuntar ciertas semejanzas, sobre todo en la industria ósea, entre el Solutrense reciente y el Badeguliense.

\subsection{Magdaleniense arcaico dividido en tres fases (Utrilla 1996, 2004)}

P. Utrilla considera que el Magdaleniense arcaico es similar, tipológica y cronológicamente, al Magdaleniense I de Le Placard y Laugerie Haute, y que se trata de un periodo que hace su presencia durante la fase Cantábrico II (GS-2c) de la periodización de M. Hoyos (1995) dándole un marco cronológico situado entre 16800 y 16000 BP (Utrilla 2004). De este modo, describe las siguientes 3 etapas para el comienzo del Magdaleniense en la región: una primera, más antigua, y dos sucesivas con carácter de facies, tanto funcionales, como culturales; aunque para otros autores, a veces estas "facies" parecen fundirse (Sauvet et al. 2008).

1. Macrolítica con muescas y denticulados. Esta etapa se correspondería con el inicio del Magdaleniense en el Cantábrico. Sería equiparable al Badeguliense (Magdaleniense I) o al concepto de Solutrense terminal ${ }^{2}$, que esta autora engloba bajo una misma identificación tipológica y cronológica. Se caracteriza por la presencia de toscas piezas macrolíticas "musteroides", el empleo de materias primas locales y elevados porcentajes de muescas y denticulados. En este grupo incluye el Mirón VR-117 y Urtiaga $\mathrm{F}+\mathrm{G}$ (en realidad no incluye los dos niveles, sólo el techo de $\mathrm{G}$, con industrias "poco características" que es difícil separar de la base de F). También incluye

(2) Este es un ejemplo de cómo casi todos los autores han visto el mismo problema, han identificado una serie de peculiaridades en el periodo transicional Solutrense-Magdaleniense, pero lo han analizado bajo diferente perspectiva, utilizando denominaciones distintas para los mismos niveles y para el mismo problema. 
La Riera 17, Chufín 1 y Amalda IV; aunque las dataciones de estos dos últimos niveles $17420 \pm 200 \mathrm{BP}$ y $17580 \pm 440$ BP respectivamente, se escapan del marco planteado por P. Utrilla (2004). Tras esta etapa, se suceden otras dos que serían contemporáneas, por lo que cobrarían un sentido pleno del término "facies" tradicionalmente empleado en el Magdaleniense cantábrico.

2a. Niveles con presencia de raclettes. En esta facies describe como características propias de la misma, tanto la presencia de raclettes, como la técnica pseudoexcisa en la industria ósea. Incluye en la misma los niveles III de Aitzbitarte IV, 5-3 de Las Caldas y III de Llonín, que sería el único en el que hay presencia de raclettes, técnica pseudoexcisa y azagayas tipo Placard/Rascaño 5 (UtriIla 1996, 2004).

2b. Magdaleniense arcaico tipo Rascaño 5. Lo describe como una facies con rareza de raclettes, azagayas de sección aplanada y monobisel en lengüeta con decoración en espiga, industria ósea poco elaborada (útiles de trabajo, cuñas, cinceles, etc...). Incluye los niveles Rascaño 5 y El Castillo 8 inferior. Cueto de la Mina D inferior, con dudas, al igual que Río/Lloseta y Lumentxa E-F (con presencia de azagayas tipo Placard).

\subsection{Transición in situ desde el Solutrense al Magdaleniense arcaico (Aura et al. 2012)}

Recientemente, J. E. Aura et al. (2012) han descrito el proceso de transición entre Solutrense y Magdaleniense en el Cantábrico, como un periodo con yacimientos afectados por graves problemas de índole tafonómica, así como por la acción de importantes procesos erosivos, realizando una primera advertencia, la confusión a la que puede llevar el diferente uso de los términos Badeguliense y Magdaleniense arcaico. Consideran que detrás subyace una cuestión historiográfica: mientras en el Levante (Parpalló) se ha preferido el uso del término Badeguliense, en el Cantábrico se ha venido usando el descriptor Magdaleniense arcaico, aunque ambos, en el contexto en el que son utilizados, deben ser considerados como sinónimos. Del mismo modo, aportan la siguiente cronología para el Cantábrico, a partir del análisis de todas las fechas disponibles, y tras expurgar todos los datos conflictivos y dudosos: Solutrense superior (23510-21490 cal BP), Magdaleniense arcaico/ Badeguliense (22290-19520 cal BP) y Magdaleniense inferior (20310-17630 cal BP).

De todos los yacimientos inmersos en este proceso, únicamente el nivel III de Llonín presenta los atributos característicos de las tres fases (presencia de raclettes, técnica pseudoexcisa, azagayas tipo Placard, abundancia del denominado utillaje "arcaico" y preferencia por las materias primas locales). Del mismo modo, indican que algunos de estos elementos también aparecen en Cueto de la Mina y La Riera, prefiriendo el uso del término "tecnocomplejos transicionales" para una serie de niveles que se encuentran a caballo entre el Solutrense superior y el Magdaleniense arcaico, y que tienen rasgos equiparables con el Badeguliense francés y el de Parpalló (Aura et al. 2012). Puede que los numerosos problemas ya relatados en los niveles cantábricos y la falta todavía de evidencias suplementarias, inviten a estos investigadores a ser cautelosos y a no decantarse por una terminología u otra, optando por una genérica denominación de "transición" para toda la problemática descrita hasta el momento en este trabajo. No obstante, sí hacen una declaración de intenciones con el análisis mostrado, que se traduce en un interesante planteamiento que viene a considerar que a pesar de que ningún conjunto o nivel es idéntico a otro en la región durante esta etapa, sí existen una serie de rasgos que son sistematizables así como una importante secuencia, que sí reúne las características presentes de manera independiente en otros yacimientos y que han dado lugar a hablar de fases o facies diferentes (Aura et al. 2012).

\section{SECUENCIAS CANTÁBRICAS}

En este punto pasamos a describir las características principales de los yacimientos y niveles objeto de debate en el marco de la transición Solutrense-Magdaleniense.

Sin lugar a dudas, uno de los yacimientos cantábricos, más importantes para referirse a la transición entre el Solutrense superior y el Magdaleniense es la cueva de Las Caldas. Esta transición está registrada en Las Caldas en la secuencia de la sala II, donde el nivel XIVc ha sido identificado como Solutrense terminal o final (17380 \pm 215 BP) y el XIII como Magdaleniense inferior (15165 \pm 160) por M.a. S. Corchón (1999). Los niveles 4 y 3 en la sala I también se corresponden con el Solutrense terminal, con dataciones de $17050 \pm 290$ BP y $18250 \pm 300$ BP respectivamente. Este horizonte solutrense terminal de Las Caldas se define por la rarificación de los elementos foliáceos y la introducción de otros nuevos, o raros anteriormente, como las raclettes, triángulos, hojitas de dorsos y azagayas biseladas decoradas (Corchón 1999).

M. de la Rasilla (1994), a la hora de analizar el final del Solutrense, ha considerado que los niveles 6-3 encajarían en la definición de Solutrense desolutreanizado, a la que hemos hecho referencia en el punto anterior. Posteriormente, algunos autores han querido ver en la secuencia de transición de esta cueva, horizontes correspondientes con el Magdaleniense arcaico/Badeguliense, lo cual ha alimentado el debate en los últimos años sobre el contexto del tránsito Solutrense-Magdaleniense en el Cantábrico. Por su parte, B. 
Bosselin (2000) ha considerado en atribuir los niveles 9-7 al Solutrense superior y los niveles 6-4 al Badegouliense, y P. Utrilla, por su parte ha incluido los niveles 5-3 en el Magdaleniense arcaico con presencia de raclettes (Utrilla 1996, 2004). Estas apreciaciones han sido también compartidas, en parte por otros autores, como Sauvet et al. (2008) que consideran, según su punto de vista, que los niveles 3 (sala I) y XIVc (sala II) podrian encajar en el Magdaleniense arcaico/ Badeguliense. Más recientemente, en una sintesis sobre la "transición" cantábrica, Aura et al. (2012) han incluido los niveles 6-3 y XIVc en el Magdaleniense arcaico "Facies raclettes".

Otra de las secuencias claves para analizar los periodos solutrense y magdaleniense inferior, es la cueva de La Riera. Como se puede observar en la Tabla 1, desde las excavaciones de L. G. Straus y G. A. Clark (1986), que describieron una importante secuencia del Solutrense superior, varios autores han querido ver en los niveles atribuidos al Solutrense superior, indicios que les hacen pensar en la existencia de niveles transicionales correspondientes al Magdaleniense arcaico/ Badeguliense.

Los niveles 9-10 de La Paloma han sido recientemente atribuidos al Magdaleniense arcaico (Sauvet et al. 2008), mientras que Cova Rosa, La Lloseta, Cueto de la Mina D inferior y Rascaño 5, se clasificaron sucesivamente como Badeguliense Tipo A (Bosselin 2000) y Magdaleniense arcaico "facies Rascaño" (Aura et al. 2012).
La cueva de El Cierro, también ha sido objeto de debate en este contexto. El nivel 4 de este yacimiento, fue considerado inicialmente Solutrense superior por F. Jordá (1960), atribución corroborada por L.G. Straus (1983) tras efectuar un estudio de los materiales; posteriormente se barajó su inclusión en el Badegouliense Tipo A por Bosselin (2000) y en este mismo congreso se ha presentado una revisión de dicho nivel, cuyo resultado puede consultarse en este volumen de actas, donde se concluye con la consideración de su posible pertenencia al Magdaleniense arcaico/Badegouliense (Álvarez Alonso y Andrés en este volumen). El nivel 4 se caracteriza por un predominio de escotaduras y denticulados, notable presencia de raederas y piezas astilladas, bajo índice de raspadores y buriles, presencia testimonial de raclettes $(n=4)$ y piezas solutrenses $(n=3)$, ausencia de laminitas de dorso. El debitado mayoritario es de lascas, estando casi ausente la técnica laminar, con un empleo mayoritario de la materia prima local (<90\% de las materias identificadas son locales).

Hoy en día, parece que uno de los contextos arqueológicos más claros en los que se podría evidenciar la existencia de un horizonte transicional, es el nivel III de Llonín, considerado Magdaleniense arcaico/Badeguliense (Utrilla 2004; Sauvet et al. 2008; Aura et al. 2012), donde estarian presentes, según Aura et al. (2012) las tres facies definidas por P. Utrilla para el Magdaleniense arcaico, confluyendo los siguientes descriptores: técnica pseudoexcisa, azagayas tipo "Placard", presencia de raclettes y utillaje "arcaico".

\begin{tabular}{|c|c|c|c|c|c|c|}
\hline & $\begin{array}{c}\text { Straus y Clark } \\
1986\end{array}$ & Rasilla 1994 & $\begin{array}{c}\text { Bosselin y Djindjian } \\
1999\end{array}$ & Utrilla 2004 & Sauvert et al. 2008 & Aura et al. 2012 \\
\hline La Riera & & & \multirow{7}{*}{$\begin{array}{l}\text { Solutrense } \\
\text { reciente }\end{array}$} & & & \\
\hline 2 & \multirow{7}{*}{ Solutrense } & \multirow{7}{*}{$\begin{array}{l}\text { Solutrense } \\
\text { superior }\end{array}$} & & & & \\
\hline 3 & & & & & & \\
\hline 4 & & & & & & \\
\hline 5 & & & & & Solutrense & \\
\hline 6 & & & & & superior & \\
\hline 7 & & & & & & \\
\hline 8 & & & \multirow{9}{*}{$\begin{array}{l}\text { Badegouliense } \\
\text { cantábrico }\end{array}$} & & & \\
\hline 9 & \multirow{8}{*}{$\begin{array}{l}\text { Solutrense } \\
\text { terminal }\end{array}$} & \multirow{6}{*}{$\begin{array}{c}\text { Solutrense } \\
\text { superior } \\
\text { desolutreanizado }\end{array}$} & & & & \\
\hline 10 & & & & & & \\
\hline 11 & & & & & & \\
\hline 12 & & & & & & \\
\hline 13 & & & & & Magdaleniense & Magdaleniense \\
\hline 14 & & & & & arcaico / & \\
\hline 15 & & \multirow{3}{*}{ Magdaleniense } & & & Badegouliense & \\
\hline 16 & & & & & & \\
\hline 17 & \multirow{4}{*}{$\begin{array}{l}\text { Magdaleniense } \\
\text { inferior }\end{array}$} & & \multirow{4}{*}{$\begin{array}{l}\text { Magdaleniense } \\
\text { inferior }\end{array}$} & \multirow{2}{*}{$\begin{array}{l}\text { Magdaleniense } \\
\text { arcaico }\end{array}$} & & \\
\hline 18 & & & & & & \\
\hline 19 & & & & & & \\
\hline 20 & & & & & & \\
\hline
\end{tabular}

A Tabla 1. Clasificación de los distintos niveles de la secuencia solutrense de La Riera, según distintos autores. 
Otro de los yacimientos con excavaciones modernas y una secuencia amplia es El Mirón. Los niveles 117 (17050 \pm 140 BP) y 313 de esta cueva, han sido descritos por Straus y González Morales (2012) como un horizonte intermedio entre el Solutrense terminal y el Magdaleniense inferior, caracterizado por toscas piezas macrolíticas "musteroides", elaboradas con materias primas locales, con elevados porcentajes de muescas y denticulados (Straus y González Morales 2005). Posteriormente, este horizonte ha sido incluido en el Magdaleniense arcaico (Utrilla 2004; Sauvet et al. 2008; Aura et al. 2012), considerando esta denominación equiparable a Badeguliense.

En cuanto a las secuencias clásicas, tenemos la importante secuencia de El Castillo, excavada a principios de siglo y, desde 1981, por V. Cabrera y F. Bernaldo de Quirós. El nivel 8 (Magdaleniense beta) de esta cueva, fue analizado por V. Cabrera (1984), quien al referirse a la presencia de piezas de sustrato en el nivel 8, indica "el sustrato siempre se ha mantenido fuerte en las colecciones de El Castillo, pero en este nivel Magdaleniense asombra el número de esquirladas que no aparecen en casi ninguna estadística del Magdaleniense inferior" (Cabrera 1984: 308); en cuanto a las reclettes hace otra interesante valoración "contamos con cuatro de estas piezas características del Magdaleniense I francés, y que en opinión de Pilar Utrilla pudieran haber sido indicio de la existencia de un nivel más antiguo, si hubieran aparecido en cantidad más representativa" (Cabrera 1984: 312). Este nivel procede de la excavación antigua de Obermaier, con lo cual la gran potencia del mismo hace que las posibles subdivisiones internas no hayan dejado constancia, por lo que se trata de un nivel en el que pudieran existir mezclas de horizontes, o la "compresión" de sucesivas ocupaciones, tal vez con matices tecnológicos y culturales importantes pero que generan un contexto estratigráfico artificial, en el que se está diseminando toda posible sucesión cultural en un gran paquete homogéneo. Estas apreciaciones fueron indicadas en su momento por V. Cabrera (1984) y P. Utrilla (1981).

El nivel 8 inferior ( $16850 \pm 220 \mathrm{BP}$ ) ha sido considerado Badeguliense Tipo A por Bosselin (2000) y Magdaleniense arcaico tipo Rascaño (Utrilla 2004; Sauvet et al. 2008; Aura et al. 2012), siendo equiparado en las dos últimas publicaciones al Badeguliense. Aunque debemos ser conscientes que debido al origen de este nivel (una excavación antigua, con metodología no sistemática), según nuestra opinión, no debe ser tomado como el mejor ejemplo para caracterizar este horizonte.

Otros niveles en debate son, Urtiaga F+G, Aitzbitarte IV (II), fechado en $16200 \pm 240$ BP y $16090 \pm 240$ BP, Lumentxa Fy el nivel 1 de Chufín, datado en $17580 \pm 440 \mathrm{BP}$, que han sido incluidos en el horizonte Magdaleniense cantábrico arcaico definido por P. Utrilla (2004), y que coincide en gran medida con las asignaciones que se pueden encontrar en Sauvet et al. (2008) y Aura et al. (2012).

\section{SÍNTESIS Y CONCLUSIONES}

Por nuestra parte, pensamos que a veces se tiende hacia una búsqueda constante de la sistematización tipológica, sin considerar la versatilidad, variedad y funcionalidad de los sitios culturalmente homogéneos e incluso en ocasiones contemporáneos, que se definen por caracteres comunes (que no idénticos). P. Utrilla $(1994,2004)$ en este sentido ya ha recalcado la importancia de las funcionalidades en el peso y diversidad de los componentes tipológicos principales, 0 característicos de un determinado conjunto lítico o nivel. En este sentido, las facies con carácter funcional, a la hora de analizar la territorialidad y adaptación de los grupos humanos del Paleolítico superior al territorio cantábrico, parece una cuestión aceptada, dentro de la que se han sugerido interesantes planteamientos (Moure 1994; Utrilla 1994). En lo que respecta a la transición entre Solutrense y Magdaleniense, creemos que no resulta un error considerar que, en un largo proceso de aproximadamente 2000 años, desde el final del Solutrense, y seguramente influenciado por un aporte exterior, ajeno a la tradición local, se generan una serie de restos en distintas cuevas cantábricas fruto de actividades, asentamientos y funcionalidades diversas y variadas en un proceso adaptativo muy versátil y de larga duración. Pensar que esto puede resolverse con la designación de un nombre 0 la simple comparación entre niveles aislados, y a veces poco equiparables a pesar de las similitudes, no parece apropiado. Es cierto que existe una cierta homogeneidad en el proceso de transición, independientemente de que le llamemos Badeguliense, Magdaleniense arcaico o Solutrense terminal. También parece evidente una larga duración y una variedad tipológica que puede responder a una diversidad funcional que se manifiesta en yacimientos con abundancia de raclettes (p.e. Las Caldas) y yacimientos con escasez de estas piezas (p.e. Rascaño).

Por otra parte, parece claro que hay ciertos paralelismos entre algunas industrias del Cantábrico y el horizonte badeguliense de Parpalló (Aura et al. 2012), como ha sido también puesto de manifiesto por P. Utrilla et al. (2012), quien llega incluso a plantear un influjo del Badeguliense levantino hacia el Cantábrico (vía valle del Ebro) apoyado en los resultados del yacimiento de Gato II, considerando por tanto el factor migratorio de aculturación como un elemento a tener en cuenta en la génesis de los cambios observados en el inicio del Magdaleniense arcaico / Badeguliense de la región cantábrica.

En definitiva y siguiendo la propuesta cronoestratigráfica más reciente (Aura et al. 2012), este contexto transicional, denominado Magdaleniense arcaico cantábrico se sitúa en la horquilla cronológica comprendida entre 22290 y 19520 cal BP, a partir de una serie de contextos estratigráficos e industrias que poco a poco van definiendo este horizonte. • 


\section{BIBLIOGRAFÍA}

ALLAIN, J. y FrITSCH, R. 1967: "Le Badegoulien de I'Abri Fritsch aux Roches de Pouligny-St-Pierre (Indre)". Bulletin de la Société Préhistorique française 64 (1): 83-94.

AurA J. E. 1988: La Cova del Parpalló y el Magdaleniense de facies ibérica o mediterráneo. Propuesta de sistematización de su cultura material: industria lítica y ósea. Tesis doctoral. Universitat de València.

- 1995: El Magdaleniense Mediterráneo: la Cova del Parpalló (Gandía Valencia). Trabajos Varios 91. Servicio de Investigación Prehistórica. Valencia.

- 2007: "Badegouliens et Magdaléniens du versant méditerranéen espagnol". Bulletin de la Société Préhistorique française 104 (4): 809-824.

Aura, J. E., Tiffagom, M., Jordá Pardo, J. F., Duarte, E., Fernández De La Vega, J., Santamaría, D., Rasilla, M. De La ; Vadillo, M. y Pérez, M. 2012: "The Solutrean-Magdalenian Transition: a view from the southwest". Quaternary International 272-273:75-87.

Bodu, P., Chehmana, L. y Debout, G. 2007: "Le Badegoulien de la moitié nord de la France. Un état des connaissances". Bulletin de la Société Préhistorique française 104 (4): 661-679.

BosseuIN, B. 2000: "Le Badegoulien en Europe sud-occidentale faciès régionaux, paléonvironnement et filiations". Paleolítico da Península Ibérica. Actas do $3^{\circ}$ Congresso de Arqueología Peninsular II:363-387.

BosSELIN, B. y DJINDJIAN, F. 1988: "Un essai de structuration du Magdalénien français à partir de l'outillage lithique". Bulletin de la Société Préhistorique Française 85 (10-12): 304-331.

- 1997: "Une révision du Solutréen de Lauguerie-Haute et le problème des transitions Gravettien-Solutréen et Solutréen-Badegoulien en Aquitaine". Bulletin de la Société Préhistorique française 94 (4): 443-454.

- 1999: "Une révision de la séquence de la Riera (Asturies) et la question du Badegoulien cantabrique". Bulletin de la Société Préhistorique française 96 (2): 153-173.

BORDES, F. 1958: "Nouvelles fouilles. Laugerie-Haute Est: premiers resultats". L'Anthropologie 62: 205-244.

BREUIL, H. 1937: Les subdivisions du Paleolithique superieur et leur signification, $2^{2 \mathrm{me}}$ edit. Paris.

BREUIL, H. y SAINT-PERIER, R. 1927: Les poissons, les batraciens et les reptiles dans I'Art Quaternaire. A.I.P.H. 2. Paris.

CabreRA, V. 1984: El yacimiento de la Cueva de El Castillo (Puente Viesgo, Santander). Biblioteca Praehistorica Hispana XXII. Madrid.

CAmps, M. y ChauHan, P. 2009: Sourcebook of Paleolithic Transitions: Methods, Theories, and Interpretations. Springer. New York.

CheYNIER, A. 1939: "Le Magdalénien primitif de Badegoule: niveaux à raclettes". Bulletin de la Société Préhistorique française 36 (9): 354-396.

- 1949: Badegoule. Station solutréenne et protomagdalénienne, Archives de l'Institut de Paléontologie Humaine 23. París.

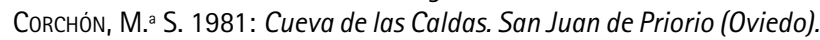
Excavaciones Arqueológicas en España 115. Madrid.

- 1994: "Arte mobiliar e industria solutrense en la Cornisa Cantábrica". Férvedes 1: 131-148.

- 1999: "Solutrense y Magdaleniense del oeste de la Cornisa Cantábrica: dataciones ${ }^{14} \mathrm{C}$ (calibradas) y marco cronológico". Zephyrvs 52: 3-32.

- 2005: "El Magdaleniense en la Cornisa Cantábrica: nuevas investigaciones y debates actuales". En N. Ferreira Bicho (ed.): O Paleolitico. Actas do IV Congresso de Arqueologia Peninsular: 15-38.

CRetin, C. 1996: "Vers une nouvelle perception du Badegoulien des Jamblancs: Premiers éléments techno-économiques". Paléo 8: 243-268.

- 2007: "Arrêt sur le Badegoulien: Historique, état de la question et perspectives". En J. Évin (ed.): Un Siècle de Construction du discours scientifique en Préhistoire, Des Idées d'hier. Actes du 26 éme Congrès Préhistorique de France, Avignon 2004, vol. 1. Société Préhistorique française. Paris: 367-378.
DUCASSE, S. 2010: La "parenthèse» badegoulienne: Fondements et statut d'une discordance industrielle au travers de l'analyse technoéconomique de plusieurs ensembles lithiques méridionaux $d u$ Dernier Maximum Glaciaire. Thèse doctorale. Université de Toulouse.

- 2012: "What is left of the Badegoulian «interlude»? New data on cultural evolution in Southern France between 23,500 and 20,500 cal. BP". Quaternary International 272-273:150-165.

DuCASSE, S. y LANGLaIS, M. 2007: "Entre Badegoulien et Magdalénien, nos cœurs balancent... Approche critique des industries lithiques du Sud de la France et du Nord-Est espagnol entre 19000 et 16500 BP". Bulletin de la Société Préhistorique française 104 (4): 771-785.

FouRLOUBEY, C. 1998: "Badegoulien et premiers temps du Magdalénien. Un essai de clarification à l'aide d'un exemple, la vallée de l'Isle en Périgord/Badegoulian and Early Magdalenian. A semantic clarification". Paléo 10:185-209.

GonzÁlez EChegaraY, J. 1960: "El Magdaleniense III en la Costa Cantábrica". B.S.A.A. 26: 1-32.

González EChegaray, J. y BarAndiarán, I. 1981: El Paleolítico superior de la cueva del Rascaño (Santander). Monografías del CIYMA 3. Santander.

Hoyos GómEZ, M. 1995: "Cronoestratigrafía del Tardiglaciar en la región cantábrica". En A. Moure y C. Gónzalez Sainz (eds.): El final del Paleolítico cantábrico. Universidad de Cantabria. Santander: 15-76.

JORDÁ CERDÁ, F. 1960: "El complejo cultural Solutrense-Magdaleniense en la región cantábrica". Primer Symposium de Prehistoria de la Península lbérica. Septiembre 1959. Diputación Foral de Navarra. Institución "Príncipe de Viana". Pamplona: 1-22.

MOURE, J. A. 1994: "Arte paleolítico y geografías sociales. Asentamiento, movilidad y agregación en el final del Paleolítico cantábrico". Complutum 5: 313-330.

RASILLA VIVES, M. DE LA 1989: "Secuencia y crono-estratigrafía del Solutrense cantábrico". Trabajos de Prehistoria 46 (1): 35-46.

- 1994: "El Solutrense en la Cornisa Cantábrica". Férvedes 1: 69-87.

- 2003: "El Solutrense cantábrico: reflexiones en torno a un diagnóstico recurrente". Lancia 5 : 33-44.

Sauvet, G., Fortea, J. Fritz, C. y Tosello, G. 2008: "Crónica de los intercambios entre los grupos humanos paleolíticos. La contribución del arte para el periodo 20000-12000 años BP". Zephyrvs LXI: 35-59.

SonNevilLe-Bordes, D. (DE) 1960: Le Paléolithique supérieur en Perigord. Burdeos.

- 1967: "Observations au sujet de la communication du Docteur Allain (23 fevrier 1967): le Badegoulien de l'abri Fritsch, aux Roches de Pouligny-Saint-Pierre". Bulletin de la Société Préhistorique Française 64: 227-229.

StRaus, L. G. 1983: El Solutrense vasco cantábrico. Una nueva perspectiva. Monografías del CIYMA 10. Santander.

Straus, L. G. y Clark, G. A. 1986: La Riera Cave. Stone Age hunter-gatherer adaptations in Northern Spain. Anthropological Research Papers 36. Ariona State University.

- 2000: "La grotte de la Riera (Asturies) et la question du Solutréen cantabrique (et Ibérique)". Bulletin de la Société Préhistorique française 97 (1): 129-132.

Straus, L. G. y González Morales, M. 2005: "El Magdaleniense de la Cueva del Miron (Ramales de la Victoria, Cantabria, Espana): Observaciones preliminares". O Paleolitico, Actas do IV Congresso de Arqueologia Peninsular. Faro: 49-62.

- 2012: "The Magdalenian settlement of the Cantabrian region (Northern Spain): The view from El Mirón cave". Quaternary International 272-273: 111-124.

Trotignon, F. 1984: "Les industries lithiques Badegouliennes". Etudes sur l'abri Fritsch (Indre), XIXè supplément à Gallia Préhistoire. CNRS. Paris: $15-100$. 
UtRILLA, P. 1981: El Magdaleniense Inferior y Medio en la Costa Cantábrica, CYMA 3. Santander.

- 1989: "El Magdaleniense inferior en la Costa Cantábrica". Le Magdalenien en Europe. La structuration du Magdalénien. Actes du CoIloque du Mayence (1987). ERAUL 38: 399-418.

- 1994: "Campamentos-base, cazaderos y santuarios. Algunos ejemplos del Paleolítico peninsular". Homenaje al Dr. Joaquín González Echegaray. Monografias del CIYMA 17: 97-113.

- 1996: "La sistematización del Magdaleniense cantábrico. Una revisión histórica de los datos". En Moure, J. A. (ed.): El hombre fósil 80 años después: 211-247.
- 2004: "Evolución histórica de las sociedades cantábricas durante el Tardiglaciar: El Magdaleniense inicial, inferior y medio (16.50013.000 BP)". Kobie (serie anejos) 8: 243-274.

Utrilla, P., Domingo, R., Montes, L., Mazo, C., Rodanés, J. M., Blasco, F. y ALDAY, A. 2012: "The Ebro Basin in NE Spain: A crossroads during the Magdalenian". Quaternary International 272-273: 88-104.

VACHERD, G. y VIGNARD, E. 1964: "Le Protomagdalénien I à raclettes des Ronces dans les Gros Monts de Nemours". Bulletin de la Société Préhistorique française 61 (1): 32-44.

VIGNARD, E. 1965: "Le Badegoulien". Bulletin de la Société Préhistorique française, Présentations et comunications 62 (8): 262-263. 
